\title{
STEM Imaging and Phase Mapping of Precipitation in Alloy 718 Using an Electron Microscope Pixel Array Detector
}

\author{
C.A. Wade ${ }^{1,2}$, E. Yucelen ${ }^{2}$, S. Sluyterman ${ }^{2,}$ B. Freitag ${ }^{2}$ and M.G. Burke ${ }^{1}$ \\ 1. Materials Performance Centre, The University of Manchester, Manchester, UK \\ 2. Thermo Fisher Scientific, Materials and Structural Analysis, Eindhoven, Netherlands
}

Alloy 718 is a Fe- and Cr-rich Ni-superalloy which may be precipitation hardened by the formation of semi-coherent the $\gamma^{\prime}$ (ordered - $\mathrm{L1}_{2}$ ) and $\gamma^{\prime}$ (ordered - $\mathrm{DO}_{22}$ ) phases in the cubic matrix $(\gamma$ ) when an appropriate heat treatment is applied. This alloy has long been of industrial significance due to its high strength at elevated temperature with excellent corrosion resistance. Cost-reducing low-loss fabrication methods for the creation of Alloy 718 parts, such as the additive manufacturing technique of selective laser melting (SLM), require the full characterization of microstructure features before these fabrication techniques can replace traditional fabrication and post process heat treatment methods. The ability to eliminate the expensive heat treatment step is dependent on a suitable distribution of $\gamma$ " and $\gamma$ ' precipitates in the matrix. In this study the distribution of $\gamma^{\prime \prime}$ and $\gamma^{\prime}$ were observed by scanning transmission electron microscopy (STEM) using the electron microscope pixel array detector (EMPAD).

The EMPAD has been developed by Cornell University and currently under commercial development with the Materials \& Structural Analysis Department of Thermo Fisher Scientific [1]. The sensor can operate from 20 to $300 \mathrm{kV}$ and consists of $128 \times 128$ pixels of 150 microns. The EMPAD records an image frame in less than a millisecond, and can detect from 1 to 10,000,000 primary electrons per pixel per second at $200 \mathrm{kV}$. These properties allow us to record the entire unsaturated diffraction pattern in scanning mode, from individual specimen locations during STEM image acquisition. This data set containing all diffraction information present over the solid angle of the detector can be used to reconstruct images by the application of a mask in reciprocal space allowing only specified information from particular angles and angular ranges to be displayed in the image.

In this study an area of 256x256 pixels on an electropolished foil of Alloy 718 fabricated by SLM was scanned with a $3 \mathrm{~nm}$ probe and a $1 \mathrm{~ms}$ dwell time. The diffraction pattern of each point in the scan was recorded using the EMPAD. The sum diffraction pattern, Figure 1a, is the sum image of the diffraction pattern from each pixel in the scan area. The reflections from $<001\rangle$ zone axis of the $\gamma$ matrix are highlighted in yellow with reflections from the [010] and [100] orientations of $\gamma$ " show in blue and red, respectively. The green reflections highlight the common reflections of both the [001] oriented $\gamma$ " and the $<001>$ orientations of $\gamma^{\prime}$. A map of phases present in the ROI, Figure 1b, shows the spatial extent and location of each of the phases present through their diffraction contrast. The dark-field image reconstructions from the $<001>\gamma$ matrix, the [001] $\gamma^{\prime} / \gamma^{\prime \prime}$, the [010] $\gamma^{\prime \prime}$, and the [100] $\gamma^{\prime \prime}$ are shown in Figure $2 \mathrm{a}, \mathrm{b}, \mathrm{c}$, and $\mathrm{d}$, respectively. In Figure 1a the choice of camera length has limited the maximum achievable angular range to a region where most scattered electrons incident on the detector still maintain coherency. Selection of shorter camera lengths allows the collection of incoherently scattered electrons to obtain Z-contrast imaging i.e. high-angle annular dark-field imaging. The combination of information from short and long camera lengths allows information from the ROI to be collected across all angular ranges in only two image acquisitions. This technique and its ability to separate information from $\gamma^{\prime}<001>$ and $\gamma$ " [001] oriented precipitates not possible through diffraction contrast alone is compared to X-ray energy dispersive spectrometry chemical maps obtained over the same ROI. 
References:

[1] M. W. Tate et al., Microsc. Microanal. 22 Issue 1 (2016), p. 237.
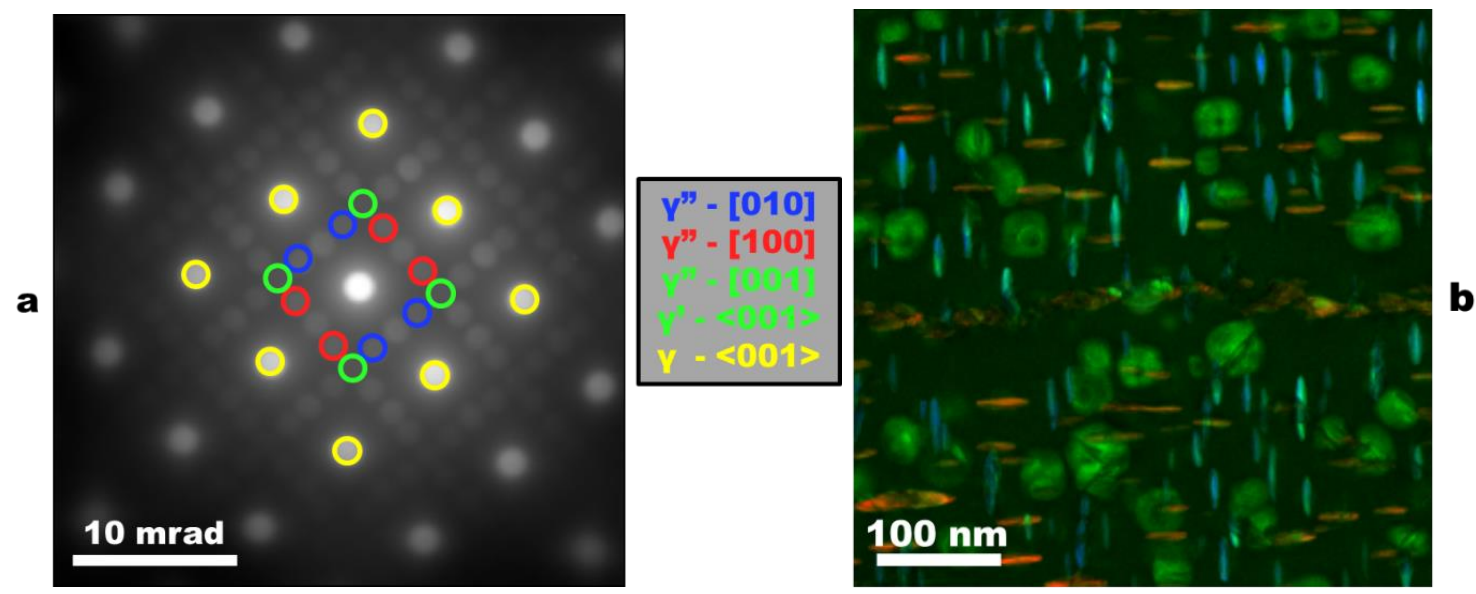

Figure 1. Sum diffraction pattern (a) of the ROI showing reflections from 3 different $\gamma$ " orientations (blue, red, and green), $\gamma^{\prime}$ (green), and $\gamma$ reflections (yellow) with combined reconstructed dark-field image phase map (b) highlighting the $\gamma^{\prime \prime}$ and $\gamma^{\prime}$ locations with legend showing precipitate / matrix direction parallel to the beam direction.

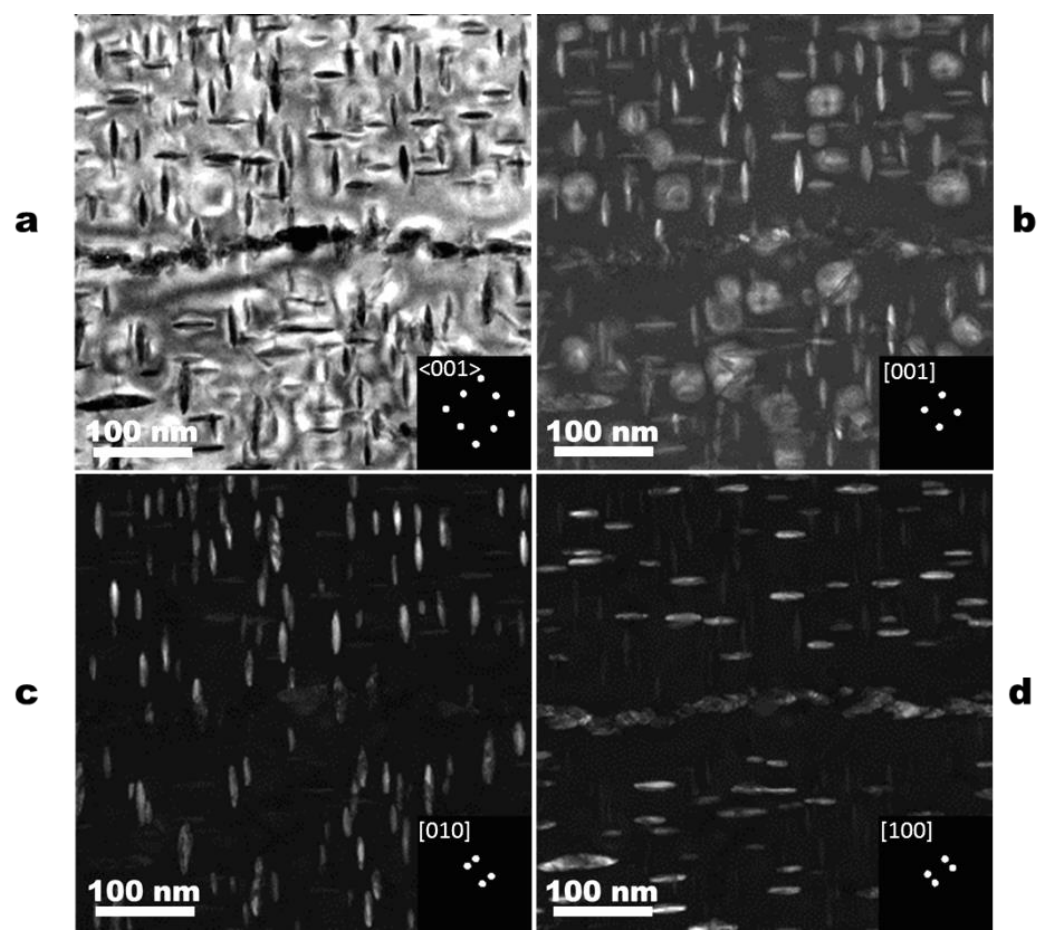

Figure 2. Reconstructed dark-field images of phases present in ROI: $\gamma$ matrix using (200) and (220) type reflections (a), $\gamma$ " and $\gamma^{\prime}$ using (110) type common reflections (b), $\gamma$ ' using (101) type reflections (c), and $\gamma$ " using (011) type reflections (d) with insets displaying mask used for reconstruction as well as precipitate / matrix direction parallel to the beam direction. 